\title{
Changing personalities: towards realistic virtual characters
}

\author{
MIKE POZNANSKI* ${ }^{*}$ and PAUL THAGARD \\ †Ontario Energy Savings Corp., Canada \\ †University of Waterloo, Canada
}

(Received 12 September 2004; in final form 31 October 2004)

\begin{abstract}
Computer modelling of personality and behaviour is becoming increasingly important in many fields of computer science and psychology. Personality and emotion-driven Believable Agents are needed in areas like human-machine interfaces, electronic advertising and, most notably, electronic entertainment. Computer models of personality can help explain personality by illustrating its underlying structure and dynamics. This work presents a neural network model of personality and personality change. The goals are to help understand personality and create more realistic and believable characters for interactive video games. The model is based largely on trait theories of personality. Behaviour in the model results from the interaction of three components: (1) personality-based predispositions for behaviour, (2) moods/emotions and (3) environmental situations. Personality develops gradually over time depending on the situations encountered. Modelling personality change produces interesting and believable virtual characters whose behaviours change in psychologically plausible ways.
\end{abstract}

\section{Introduction}

When we think of what people are like, how they generally act and behave, we usually think of their personality. Personality is very important to us, because it defines who we are. It separates our particular way of behaving from somebody else's. When we watch a movie, theatrical performance, or play a video game, we enjoy characters with different and attention-grabbing personalities. It is personality that makes the characters appealing. This article describes a new computational model of personality with personality change. Section 1 begins by defining and motivating the problem. Section 2 briefly outlines existing research related to computer modelling of personality. Section 3 describes the model presented in this

\footnotetext{
*Corresponding author. Email: mikepoznanski@yahoo.ca
} 
article in detail. Section 4 presents some sample model results along with an evaluation.

\subsection{What is personality?}

Generally, personality refers to those characteristics of a person that account for consistent patterns of behaviour, over situations and time (Pervin 1989: 4). Those characteristics include tendencies towards emotions, thoughts and actions. In this article, we focus mainly on the action or behavioural aspects of personality, but also take emotions into consideration.

\subsection{Personality in computer science}

Even a few years ago, the idea that modelling personality could be important in computer science would have sounded ridiculous. However, as computer science is rapidly expanding into new fields, there are several areas where modelling personality can be crucial. There are growing attempts to create human-like, convincing virtual characters called Believable Agents (see, for example, Trappl and Petta 1997). Increasingly such agents are made more believable by giving them emotions and personalities. One area where these agents are needed is human-machine communication and interfaces. In this setting, lifelike, personalized agents make humanmachine communication and interaction more enjoyable and easy. Another area that requires Believable Agents is electronic marketing and advertising, where personality-driven virtual sales agents or advertisers can present their products to human customers in a unique and attention-grabbing way.

Perhaps the largest and most profitable computer field where Believable Agents are needed is the electronic entertainment industry. This includes virtual reality, where virtual characters or avatars with personalities create more interesting interactive virtual worlds: virtual theatre, where virtual actors are made more believable and entertaining if they have personalities; and of course video games. The video game industry is now larger than the movie industry, at least in terms of revenues (see, for example, King 2002). According to researchers in video game artificial intelligence (AI), 'Computer game developers are starting to recognize the need for human-level artificial intelligence', and 'interactive computer games provide a rich environment for incremental research on human-level AI' (Laird and van Lent 2001: 17, 15). There is a great need for building more interesting characters for video games, and one way to do it is by giving these characters personalities. We next explain how this is already being done to some degree.

\subsection{Personality in video games}

Probably the best example so far of game characters with personalities is the bestselling PC game of all time: The Sims ${ }^{\mathrm{TM}}$ (www.TheSims.com; see, for example, 'The Sims Becomes The Best Selling PC Game of All Time', 2002). The Sims is an interactive simulation of everyday life. You play as a virtual character of your choice (a sim), go to work, start a family, make friends with other sims, build relationships, enjoy pastime activities, and other similar things. When you create your characters 


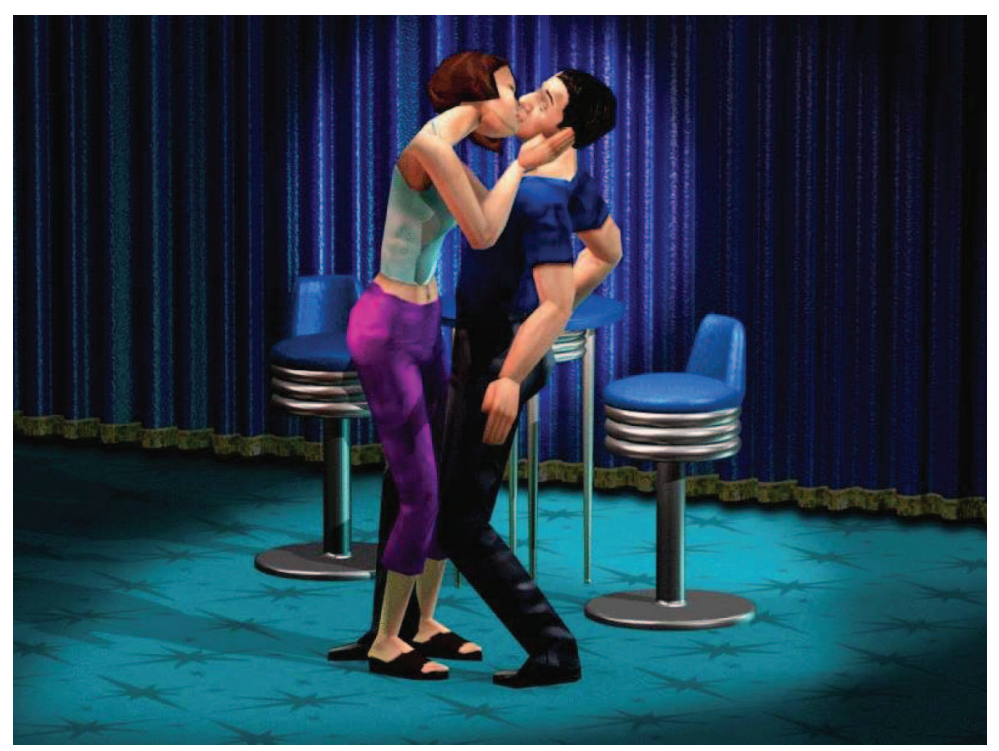

Figure 1. Playful sims (source: GameScreenshots.com, The Sims Hot Date, PC). (The Sims Hot Date (C) 2001 Electronic Arts Inc. The Sims is a trademark or registered trademark of Electronic Arts Inc. in the US and/or other countries. All rights reserved.)

at the beginning of a game, you can choose their personalities from among: Neat, Outgoing, Active, Playful or Nice (see figure 1).

A sim's personality affects how he or she behaves in the game. For example, outgoing sims make friends faster, while neat sims keep themselves and their surroundings very clean. Personality traits also affect the ability to build particular skills. For instance, an active sim will find it easier to develop the body than an inactive one. So the sims have personalities to some extent, and it is their personalities that make them more interesting and separate one sim's behaviour from another (see figure 2).

Just as the sims' personalities make their behaviour appealing and different from one another, sims with more complicated personalities would result in even more exciting and varied behaviour. So a sim with a more complex personality model would help make the game more engaging and enjoyable. We will argue later that our personality model has among other things a new feature-namely personality change-that will result in more interesting and varied behaviour of virtual characters. In order to better understand personality, and to model it computationally, we need to understand some of the major personality theories in modern psychology.

\subsection{Personality in psychology}

The major personality theories important for our discussion are: trait theories, psychobiological theories and social learning theories. A personality trait is an enduring personal characteristic that reveals itself in a particular pattern of behaviour in different situations (Carlson and Buskist 1997: 450). By analysing 


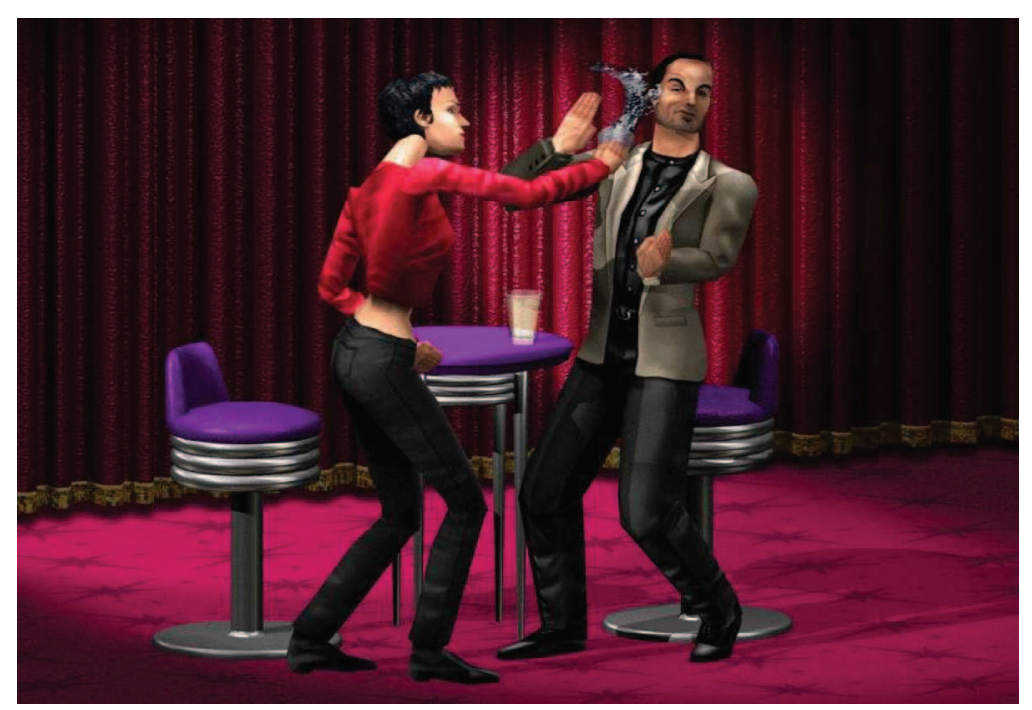

Figure 2. Not-so-nice sims (source: GameScreenshots.com, The Sims Hot Date, PC). (The Sims Hot Date (C) 2001 Electronic Arts Inc. The Sims is a trademark or registered trademark of Electronic Arts Inc. in the US and/or other countries. All rights reserved.)

behavioural tendencies, psychologists have categorized people's personalities in terms of traits. The most popular and influential of the Trait theories is the Big Five or the Five-Factor model, which claims that people's personalities can be described by five major traits: openness (to experience), conscientiousness, extroversion, agreeableness and neuroticism. These five traits can be easily remembered by the mnemonic OCEAN. People who are open to experience are curious, creative, original, imaginative, untraditional and have broad interests. Conscientious people are organized, reliable, persistent, hard-working, self-disciplined and honest. Extroverted people are sociable, active, talkative, optimistic and fun loving, as opposed to introverted people. Agreeable people, as the name might suggest, are good-natured, trusting, helpful, forgiving and straightforward, in opposite to disagreeable people. Finally, people who are neurotic worry a lot and are nervous, emotionally unstable and insecure. It is important to note that the Big Five personality model is a descriptive or categorical model, rather than an explanatory model, but it has been successfully used to predict people's behaviour based on their personality traits (Paunonen 2003).

Psychobiological theories try to explain personality through heritability and biological mechanisms. Studies indicate that various personality characteristics can be heritable, although personality is also strongly influenced by the environment. Generally, heritability is considered to be responsible for around $50 \%$ of the variability in personality traits, and the environment is considered to be responsible for the other $50 \%$. Of course, the interaction of heredity and environment is an important part of personality, which brings us to the next big theory of personality.

Social learning theories, also called behavioural-cognitive theories, state that much of one's personality is learned through interaction with the environment. Personality is influenced both by personal characteristics like personality traits, and by 
environmental situations. A person's beliefs and expectations about the consequences of their behaviour in given situations, including emotions generated by these beliefs and situations, develop the person's personality. The beliefs and expectations are about the success, and potential for reinforcement or punishment, of a person's behaviour.

It is probably true that no single one of these theories can completely explain all aspects of personality. The computational model of personality presented in this article will be largely based on the Big Five trait model, but will also have some components from the psychobiological and social learning theories, in particular the influence of the environment on personality and genetic predispositions for personality.

\subsection{Why build computational models of personality?}

We can separate the reasons for why one would want to build computer models of personality into two types: the psychological motivations and the computer science or artificial intelligence incentives. From the psychological perspective, computer models of personality and simulations conducted with them can help us better understand personality and human behaviour. For instance, Read and Miller (2002) use a computational model of personality to illustrate personality dynamics, situational responsiveness and consistency of persons in situations. A computer model has the advantages of easily simulating different personalities in different scenarios and looking at the hidden personality processing that goes on, a task that is extremely difficult in real-life personality experiments.

From the computer science point of view, computational models of personality can help create better virtual characters or Believable Agents, which can be usefully applied in a number of previously discussed areas. Ideally, we would like a model that can satisfy both the psychology and computer science goals.

\subsection{Criteria for a good computational model of personality}

We need to define some criteria that will tell us what a good computational model of personality should look like. These criteria will also help in evaluating and comparing computer personality models, as these models tend to differ in many ways, including their architectures and even model inputs and outputs. Of course, these criteria will differ depending on the motivations for the model and its applications. Nonetheless, there are a few general measures that most computational personality models should have.

The first of these is psychological plausibility and realism, and psychological explanatory power. We would like the model to adhere to and not violate any known psychological findings, as well as help us understand personality. This criterion can be broken down into psychological components that are important in personality. This includes questions such as: How much of a given theory of personality does the model cover? Does the model incorporate moods, emotions, genetics and environmental influences on personality? Does the model help explain personality?

A second general criterion is simplicity and efficiency. If the model is simple yet interesting then it is better than one that is complicated. By simplicity, we mean both simple in design and efficient in terms of running time and computational resources 
such as memory. This is particularly important for models whose applications are in video games, where computational resources should be kept to a minimum.

Finally, as a third general criterion for good computational models of personality, we would like the virtual characters who are based on these models to exhibit interesting and varied behaviour. Of course, this is a subjective criterion, but we can say that more behaviours and more varied behaviours are generally more interesting than fewer and repetitive behaviours. Behaviours that change over time are more human-like, less cyclic and hence more believable. In other words, virtual characters whose personalities (and consequently behaviours) change in psychologically plausible ways are more interesting than virtual characters whose personalities and behaviours do not change. Again, this measure is especially important for video game applications, since we want our virtual characters to be engaging and fun.

\subsection{A computational model of personality that includes personality change}

The computational model of personality presented in this article aims for both psychological plausibility and computational appeal concerning interesting behaviour of virtual characters. The principal difference between this model and other related work to date is the modelling of personality change or development. In the proposed model, personality changes in psychologically plausible ways, depending on the environmental situations encountered. Such personality change results in more believable and interesting virtual characters. This work is related to adaptive artificial intelligence, in the sense that the virtual characters modelled adapt or change their behaviour in the model. The model was designed with an AI game application in mind, similar to The Sims for example, although it could be used for other applications that require virtual personalities.

\section{Related work}

Recent years have shown increasing efforts at emotion and personality modelling, both by psychologists and computer scientists. On the psychology side, Read and Miller (2002) have built a recurrent neural network model of personality that is primarily trait and goal oriented, as the authors believe that most personality traits are fundamentally goal based. Shoda and Mischel (1998, 2002) consider personality to be a dynamical system, viewed as a cognitive-affective processing system (CAPS), that functions as a parallel constraint satisfaction network. A personality is modelled through interconnected cognitive-affective units (person variables) that include beliefs, goals, emotions, expectations, memories and various skills important for self-regulation.

On the computer science front, Mac Namee and Cunningham (2002) propose a neural network model for social interaction created for a Sims-type video game. Their model consists of a trait-based personality component, and attribute-based mood and relationship components. Kshirsagar and Magnenat-Thalmann (2002) have built a multi-layer personality model, based on the Big Five theory, using Bayesian networks. Their goal is to create virtual humans that can interact spontaneously using natural language, emotions and gestures. In the Virtual Theatre project, Rousseau and Hayes-Roth $(1996,1997)$ build personalities for virtual actors 
that portray fictive characters by improvising their behaviour in a multimedia environment. Their personality model is rule-based, and derived from trait theories and social learning theories. Moffat (1997) presents a heavily emotion-based personality model for an autonomous AI agent. His model is largely based on Frijda's (1986) theory of emotions, which says that emotions arise as a result of appraising stimuli or events, compared to an individual's concerns. The Oz project (Bates et al. 1992, Loyall and Bates 1997) aims to build believable goal and emotion driven autonomous agents that communicate through natural language, for virtual entertainment. The personality-relevant parts of these agent's architectures are composed of a goal-directed action engine and an event-appraisal model of emotions based on the work of Ortony et al. (1988). Andre et al. (1999, 2000) have built computer models of emotions and personality for children's virtual puppet theatres, virtual sales presentations and virtual guides for Internet websites. Rizzo et al. (1998) model personalities of Believable Agents using goals with different priorities and goal-based preferences over actions and plans used for achieving those goals.

None of the above computational models of personality simulate personality change. Other neural network models that integrate cognition and emotions include Nerb and Spada (2001), Thagard (2003) and Wagar and Thagard (2004).

\section{Model description}

We will call our personality model SPOT (simulating personality over time). The model description is divided into two parts: (1) personality modelling without personality change and (2) modelling personality change over time. The first two subsections deal with part (1), while the following two subsections describe part (2).

\subsection{Overview of SPOT}

SPOT is psychologically based and intended to be used as an application in a Sims-type video game. SPOT has four basic components: personality, emotions, inputs describing the situations and behaviour outputs (figure 3). The arrows indicate influences or relationships between the components.

The personality component of SPOT is based on the Big Five trait model, and allows one to specify a personality consisting of any combination of the Big Five traits (open, conscientious, extrovert/introvert, agreeable/disagreeable, neurotic). The emotion component consists of six basic emotions: neutral, sad, happy, angry, afraid and curious. The inputs consist of three parts: situation factors describing the current situation, relationship factors describing the relationship to another character and mood specifying the current mood of the character modelled. The outputs consist of behaviours or actions that the modelled character can engage in. As can be seen from the diagram, output behaviours are influenced by the input situation and relationship factors, the character's personality, and the character's emotions. Emotions in turn are influenced by the character's personality, as well as the input situation, relationship and mood factors. Thus, the personality of a character and his or her behaviour results from the interplay of three components: personality traits, emotions (and moods), and situational factors. Let us now look at SPOT in more detail. 


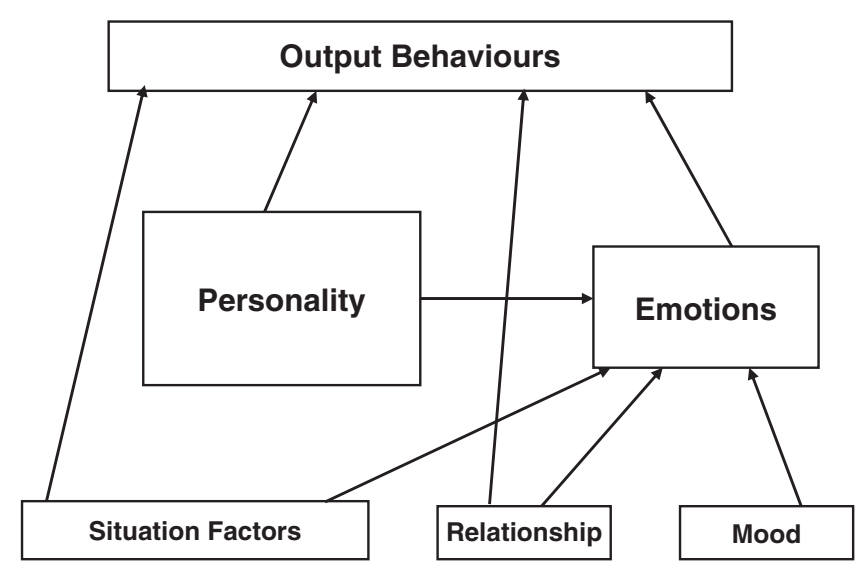

Figure 3. Overview of the SPOT personality model.

\subsection{Personality and behaviour modelling}

SPOT is implemented as a neural network, using JavaNNS (version 1.1), a freely available, powerful neural network simulator (JavaNNS 2003). JavaNNS was developed at the University of Tubingen, and is based on the Stuttgart Neural Network Simulator (SNNS) developed at the University of Stuttgart. Below is a screenshot of SPOT as implemented in JavaNNS (figure 4). SPOT is a simple threelayer, feedforward neural network. Each of the 34 nodes in the network represents a particular feature of SPOT, such as a specific emotion.

There are seven different input situations in the situational feature part of SPOT. A neutral situation (s_Neutral) is one where nothing exceptional is happening, for example being in a calm crowd of bystanders. A friendly situation (s_Friendly) is one where the character is being treated nicely, for example being in a group of close friends. A hostile situation (s_Hostile) is the opposite, where a character is being treated rudely, violently or is in immediate danger. A help situation (s_Help) is one where help is required, for example finding someone who needs a hand with some task. An explore situation (s_Explore) requires the character to explore his surroundings, for example investigating a new area he is in. A persist situation (s_Persist) requires the character to persist at some task. Finally, a stressful situation (s_Stress) is one where the character is exposed to some environmental stressors, for example being harassed by your boss at work.

The relationship part of the input layer is used when there is another character available for interaction. It consists of two nodes, $r_{-}$Like and r_Dislike, and specifies how much the modeled character likes or dislikes the other character available for interaction. The mood part of the input layer specifies the current mood of the modelled character. It can take on three values: neutral, good and bad mood (m_Neutral, m_Good, and m_Bad). The output layer consists of nine different behaviours or actions that a character can engage in. The input situations and output behaviours were chosen to be representative of the Big Five personality traits modelled, and are sufficiently diverse to allow game characters to respond differently to various situations. 


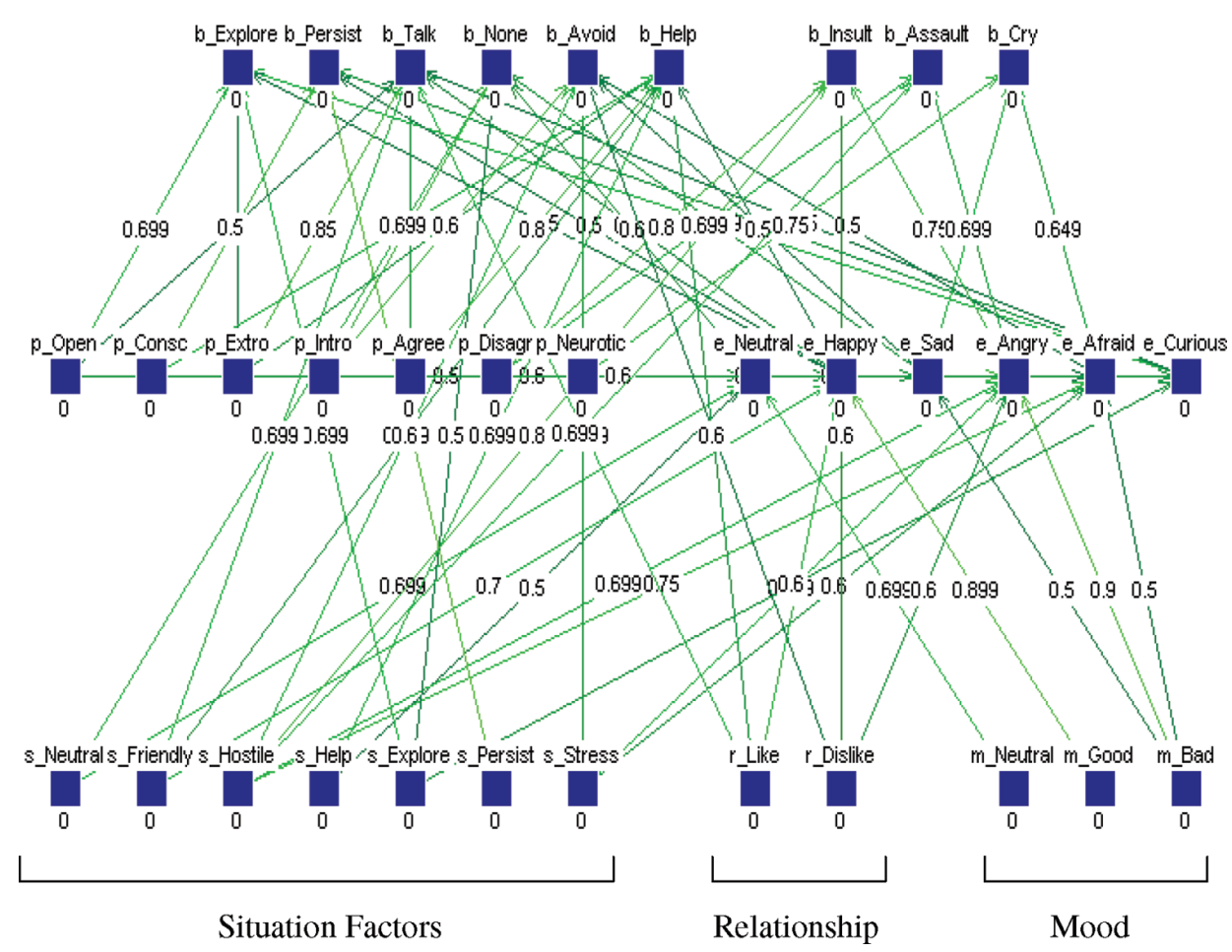

Figure 4. The SPOT personality model as implemented in JavaNNS. (Links weaker in weight than 0.5 have been omitted for clarity.)

The emotion component of SPOT is in the middle layer of the network. Emotions are influenced by situational factors, moods and personality traits. The personality component of SPOT contains nodes for the Big Five personality traits. These personality nodes are really part of the input layer, as they contain no input connections. A particular personality is specified by setting one or more of these personality nodes to some desired values. Values can range from 0 to 1 , representing the strength of that component. (A value of 0 actually means that that component is not specified, or not being used. For example, a value of 1 for p_Open means that the current character is extremely open, a value of 0.5 means that they are somewhat open, a value of 0.1 means that they are not very open, while a value of 0 means that we do not know or do not wish to specify that component.) Input situations as well as mood and relationship input values are specified the same way.

According to the Big Five personality theory, each of the Big Five traits has behavioural tendencies or predispositions characteristic for that trait. Therefore, the Big Five personality nodes in SPOT are connected to output behaviours according to the tendencies of a given personality trait for a particular behaviour. Thus, the extrovert node (p_Extro) is connected to behaviours characteristic of extroverts, such as talking (b_Talk) and helping (b_Help), although it is also connected to other non-extrovert behaviour nodes such as doing nothing (b_None) and exploring (b_Explore), to allow for other behaviour possibilities. Similarly the other 
personality nodes are connected to behaviours characteristic of those traits, as well as a few other non-characteristic behaviours. The strength of a connection can range from 0 to 1 , and represents the influence of the source node on the target node. Thus the connections from the extrovert personality node to the behaviour nodes b_Talk, b_Help, b_None and b_Explore are $0.85,0.6,0.3$ and 0.5 respectively. This means that someone who is extroverted is more likely to engage in extroverted behaviours such as talking and helping, and less likely to engage in other non-extroverted behaviours such as doing nothing.

The other personality nodes are connected to their characteristic behaviours in the same way. Thus, an open person is more likely to explore, while a conscientious person is more likely to persist and help. An introvert is more likely to avoid or do nothing. An agreeable person is more likely to help or talk, while a disagreeable person is more likely to insult or assault others. Finally, a neurotic person is more likely to cry and avoid by nature. As another illustration, the disagreeable personality node (p_Disagr) has connections to output behaviours b_Insult, b_Assault and b_Avoid, with strengths 0.8, 0.7 and 0.3, meaning that a disagreeable person is more likely to insult, assault and avoid others by nature.

Specifying values for the personality nodes can be considered as giving the person or character genetic predispositions for personality traits, as in the psychobiological theory of personality. This means that a person will be predisposed genetically to engage in certain characteristic behaviours more likely than in others. On top of the Big Five tendencies and psychobiological genetic predispositions for behaviour, there are additional ways in which personality and behaviour are determined in SPOT: situations, as well as moods and emotions also affect behaviour.

Social-Learning theories of personality tell us that behaviour is influenced by particular situations encountered. Therefore, each situation in the input layer connects to the corresponding behaviour in the output layer, as well as some other behaviours for variety. Thus, a neutral situation will have the strongest connection to the b_None output node ( 0.7 strength), but it will also connect to the b_Avoid and b_Talk nodes with lesser strength ( 0.2 and 0.4 respectively). Similarly, a friendly situation influences the talk and help behaviours most strongly, while a hostile situation influences the insult, assault and avoid behaviours the strongest. Likewise, the help situation influences the help behaviour, the explore situation affects the explore behaviour, the persist situation influences the persist behaviour, and the stress situation influences the avoid and insult behaviours. Additionally, the r_Like and r_Dislike relationship nodes similarly influence appropriate behaviours (like influences talk and help, while dislike influences avoid, insult and assault).

As depicted in figure 3, emotions in SPOT are influenced by the character's personality, as well as by situation, relationship and mood factors. Therefore, the six possible emotions in the middle emotion layer have input connections from the personality, situation, relationship and mood components. The personalityto-emotion connections specify how likely that particular personality trait will experience that particular emotion. Thus an open-minded person will be curious by nature (0.6 strength), extroverts and agreeable people will be somewhat happy (0.6 strength), disagreeable persons are mostly angry (0.8 strength), 
while emotionally unbalanced neurotic people are somewhat sad and afraid (0.6 and 0.5 strengths).

Situation, relationship and mood factors activate emotions that correspond to that particular situation, relationship or mood factor. For example, in a neutral situation a person will feel largely neutral, a friendly situation will make them feel happy, while a hostile situation will make them feel somewhat angry and afraid. A character who likes another character will feel rather happy, whereas a character who dislikes another character will experience negative emotions such as anger and fear. Similarly, someone in a neutral mood will feel mainly neutral, a person in a good mood will generally feel happy, while a character in a bad mood will feel mostly angry.

In much the same way as with personality-to-behaviour connections, particular emotions influence output behaviours appropriate to those emotions. In general, positive emotions will produce positive behaviours, and negative emotions will produce negative behaviours. Thus, the happy emotion tends to produce positive behaviours such as talking and helping, while an angry emotion will tend to produce negative behaviours such as insulting and assaulting. In this manner, situation, relationship and mood factors activate emotions appropriate to the particular circumstances, which in turn influences behaviours appropriate for those emotions.

Connections between nodes and their strengths were determined based on known behavioural and emotional tendencies of the Big Five personality traits, and common sense about human behaviour, personality and emotions. The connections and weights were subsequently refined as necessary, by conducting numerous model simulations. These simulations used various personalities, situations, relationship factors and moods to make sure the output behaviours are appropriate given the inputs. For example, a highly neurotic (1) personality, in a stressful situation (1) and a bad mood (1) should do something emotionally unstable, like cry. (For other examples of similar test simulations, please see table 2 in section 4.1.) The model is not very sensitive to changes in the connection weights; that is, small changes in the connection weights generally result in small changes in the model output.

Such hardcoding of connections and weights was chosen over training the network for two main reasons. First, it would have been extremely difficult to create a large enough training set for this network, as there are no data sets available for human behaviour. Mac Namee and Cunningham (2002) also point out this problem with their neural network model, and the difficulties they had with it. Second, if the network were to be modified even slightly, by say adding another input or output node, a new and much larger training set would be required. In SPOT, adding a new node is not very difficult at all: one just inserts the new node, along with its appropriate connections and weights to the other nodes. The rest of SPOT remains unchanged, and no training is necessary.

Moreover, whether manually setting up the connections and weights or creating a data set for training the network, subjectivity in the corresponding input/output determination is inescapable. In fact, all of the personality models reviewed in this article require some subjectivity in creating the model, either producing the neural network training set in the $\mu$-SIC model, estimating transition probabilities in the multilayer personality model, or specifying personality and behaviour rules in the Virtual Theatre, Will or Oz project models. 
Another way of looking at SPOT and interpreting the way connections are set up is by analogy with rules. The connections between different nodes can be thought of as representing rules regarding specific situations, moods, personality traits and behaviours. For instance, if we have someone who is disagreeable, in a hostile situation and in bad mood, then they should behave in an unpleasant way. This rule is represented in the network by having connections from disagreeable personality to disagreeable behaviours, from hostile situation to disagreeable behaviours, and from bad mood to angry emotion, which in turn also connects to disagreeable behaviours. In this sense, the network represents a large number of such rules, but in a much more flexible manner. The rules do not have to be specified explicitly, but are rather encoded in the connections between nodes and their strengths. This makes it easy to represent a large number of rules in a concise fashion.

Once a particular personality has been set up, by specifying values for the personality nodes, and a combination of situation, relationship, and mood factors has been given, activation flows in the network feedforward style from the input situation layer, through the middle layer, to the output behaviour layer. The activation function used for every node is simply the sum of all input connection weights multiplied by their nodes' activations. That is, the activation of unit $j$ is $a_{j}=\sum_{i=1}^{N} w_{i j} * a_{i}$, where $w_{i j}$ is the weight of the input connection from unit $i$ to unit $j$, $a_{i}$ is the activation of input unit $i$, and $N$ is the number of input units connected to unit $j$. The updating of node activations is a simple one-time process. Input nodes that are not being used (in a given simulation) are assigned zero values, which means that they do not contribute anything to the network's activation. There are no inhibitory connections in SPOT, which means that no network settling is required, resulting in fast execution. (Inhibitory connections were deemed not necessary, because SPOT is not a winner-take-all model. Multiple nodes can be activated in the model at the same time, but only the most activated output node determines choice of behaviour.)

Currently the output behaviour selected by the character is the behaviour corresponding to the most activated output node. However, to introduce more behaviour variety in SPOT, one could probabilistically choose from the three most activated output nodes, selecting the most activated behaviour most of the time, and the other two runner up behaviours less frequently.

In the following subsections, we describe how personality change or development is included in SPOT. We begin by motivating the need and reasons for personality change using research from psychology.

\subsection{Can personality change?}

Recent evidence in psychology suggests that personality can indeed change throughout life and that the environment can influence personality. Heatherton and Weinberger (1994) present a comprehensive review of influential personality theories and their views on personality change. They conclude that there seems to be general agreement among researchers that personality can and does change through about age 30 (Heatherton and Weinberger 1994: 335). Loehlin (1992) analyses the effects of genes and environment on personality development in a number of important studies. He concludes that longitudinal studies of personality change in adolescence 
and adulthood suggest that personality changes do occur, and furthermore, that most of these changes might be due to environmental effects (Loehlin 1992: 104). Srivastava et al. (2003) surveyed over 130000 adults aged 21-60 on the Big Five personality traits. They found that personality changes even beyond age 30 , and that average levels of personality traits change gradually but systematically throughout the lifespan.

Even though there seems to be growing agreement that personality does change throughout life, and that the environment can influence personality change, it is still unclear how exactly this happens. Research with children shows that young children who are cared for and loved by their mothers tend to be most popular and sociable later in school. Conversely, young children who are not cared for particularly well and are ignored, have difficulties with social adjustment later in childhood: they have poor social skills and tend to be hostile, impulsive and withdrawn (Carlson and Buskist 1997: 391). Disagreeableness seems to have a large environmental influence as well: parents who beat their children usually turn out to have been victims of child abuse themselves (Carlson and Buskist 1997: 431). Abusive parenting is also considered a possible influence in the development of antisocial personality disorder (Carlson and Buskist 1997: 582).

Vernon et al. (1997) tried to identify environmental predictors of differential personality development in twins and non-twin siblings. Environmental factors were linked to personality trait development similar to the Big Five. The study found that, for non-twin siblings, conscientiousness differences correlated with differences in environmental factors termed 'sensation-seeking technology-interests' (seeking activity; having technology interests) and with parental affection and control. Differences in openness correlated with differences in the 'sensation-seeking technology-interests' environmental factors. Finally, differences in neuroticism correlated with differences in family environment acceptance-rejection (did the person accept or reject their family environment?).

This research shows that personality can and does change, and it also sheds some light on how environmental factors might influence personality change. Therefore, to better understand and model personality, we would want to include personality change in a computational model of personality. However, there are also good reasons why we would want to model personality change with computational or video game applications in mind. First, we would like our characters to be as humanlike as possible, and that includes changing or learning. Furthermore, a virtual character whose personality changes will exhibit more interesting and varied behaviour over time. A sim-type game character whose personality remains the same will always exhibit the same (or very similar) behaviour in identical situations. However, the same character with a changing personality could behave quite differently in identical situations over time, resulting in less predictable and more believable behaviour.

\subsection{Modelling personality change over time}

Based on the above research, we can set up some general rules for how the environment influences personality change. First of all, drastic personality change is uncommon, and normally requires extreme or unusual circumstances, such as far-reaching personal experiences or disasters. Therefore, we will model 
Table 1. Summary of personality change rules in SPOT. Each situation encounter has the corresponding effects on personality.

\begin{tabular}{ll}
\hline Situation encounter & \multicolumn{1}{c}{ Effects on personality } \\
\hline Friendly & $\begin{array}{l}\text { more Extroverted AND more Agreeable } \\
\text { less Introverted AND less Disagreeable }\end{array}$ \\
Hostile & IF Disagreeable THEN more Disagreeable \\
& IF Introverted THEN more Introverted \\
& ELSE randomly more Disagreeable OR more Introverted \\
& ALSO less Extroverted AND less Agreeable \\
Explore & more Open \\
Persist & more Conscientious \\
Stressful & more Neurotic
\end{tabular}

personality change as a gradual process. Personality will change due to environmental influences from different situations. That is, whenever a person encounters a particular situation, that situation will change his or her personality slightly. Which personality traits are changed will depend on the situation.

Given the findings above, one can say that affection and caring tend to make people more social and nicer. Therefore, in SPOT, friendly situations will tend to make people slightly more extroverted and more agreeable, while at the same time making them slightly less introverted and less disagreeable. Lack of caring and hostility appear to make people less social and more aggressive. For this reason hostile situations in SPOT will tend to make people more introverted and more disagreeable. To make this rule more interesting and psychologically plausible, someone who is introverted will become more introverted after a hostile encounter, while someone who is disagreeable will become more disagreeable. All other personalities will randomly become either more introverted or more disagreeable after a hostile encounter. Furthermore, a hostile situation will also make every personality less extroverted and less agreeable.

'Sensation-seeking' appears to correlate with the trait of openness. Therefore, situations where one is required to explore in the model will make people more open. Neuroticism correlates with family environment acceptance-rejection. Because rejection can be a stressful experience, in the model stressful situations will make people more neurotic. Situations requiring someone to persist at some task will tend to make people more persistent. These personality change rules are summarized in table 1 .

In this way, the environment changes personality over time. However, as previously discussed, personality also has genetic components, predisposing people to behave in certain ways. Therefore, we would not want to ignore these genetic predispositions and allow the environment to change someone into a completely different person. For example, we would not expect someone who is extremely disagreeable to change into someone who is completely agreeable as a result of encountering many friendly situations. For this reason we will impose limits on how much personality can change due to the environment. Once this limit has been reached, the environment will not be able to change personality any more. 
This gives us some very simple yet psychologically inspired rules for how the environment shapes personality change over time. Each situation encounter changes personality by a very small amount. In the long run, however, these small changes can accumulate and considerably change someone's personality, but only up to some limit. For example, someone who is repeatedly being treated in a hostile manner will become more disagreeable over time. Similarly, someone who experiences stressful situations consistently will become more neurotic as time progresses.

The degree to which each situation changes the corresponding personality node depends on whether the modelled personality was initially (genetically) extreme or not. Currently a personality trait is considered to be extreme if the corresponding personality node value is greater than 0.8 or less than 0.2 . If someone is nonextreme in any personality trait to begin with (that is in the range [0.2-0.8]), they always change by a fixed amount in that trait, with every situation. This fixed amount is pre-specified at the beginning of the simulations. Currently this value is set to 0.05 .

On the other hand, extreme personalities change more slowly initially with every situation, compared to non-extreme personalities. This is because extreme personalities are considered to be more resilient to change than non-extreme personalities. We would expect someone who is extremely disagreeable initially, to change more slowly than someone who is only somewhat disagreeable to begin with. For this reason extreme personalities change very slowly at first, and then progressively faster with each situation, until they change by the same amount as non-extreme personalities. The change amount is currently 0.005 for the first situation encounter, and increases gradually by 0.005 with each subsequent situation, until the change value for non-extreme personalities is reached (currently 0.05). These change amounts increase independently for each personality trait, since different situations affect different traits.

The imposed limits on how much personality can change also depend on whether the modelled personality was initially (genetically) extreme or not. If someone is initially extreme in any personality trait, they cannot change beyond a value of 0.5 in that trait. Thus, someone who starts off being 0.9 extroverted will not be able to go below 0.5 in that trait, and someone who starts off being 0.1 agreeable will not be able to go above 0.5 in that trait. People who start off non-extreme in any personality trait cannot change to the extremes in that trait. Hence, someone who starts off being 0.3 open initially will not be able to change below 0.2 or above 0.8 in that trait. By imposing personality change limits in this manner, we do not violate the person's initial genetic personality predispositions, nor do we allow the environment to change someone into someone completely different.

The rate of personality change per situation encounter, as well as the imposed personality change limits, were chosen to be psychologically reasonable and interesting in terms of gaming applications. If necessary or desired, these values may be easily changed. Therefore, for example, if we wanted personality to change faster or slower in our game, we could increase or decrease the personality change parameter. The imposed limits on personality change can similarly be increased or decreased. Furthermore, the personality change rules are also easily modifiable. For instance, we might want hostile situations to increase disagreeableness regardless of whether the person was disagreeable or introverted to begin with. This modification is simply a matter of adding or removing a few if-statements in the personality change program. 


\section{Results}

This section provides a few sample simulations illustrating SPOT's performance.

\subsection{Clearly distinguishable personalities do things characteristic of their traits}

Table 2 below presents seven simulations that show that SPOT produces characteristic behaviours for each one of the Big Five traits. The numbers in brackets indicate the activation value of the corresponding node in the neural network model.

An extrovert (1) in a neutral situation (1) and in a neutral mood (1) should be extroverted. Accordingly, the model result is a talk behaviour (2.14). Conversely, an introvert (1) in a neutral situation (1) and a neutral mood (1) should be introverted. The model output is b_None (2.7), followed by b_Avoid (1.6), which are behaviours characteristic of introverts. An agreeable person (1) in a situation which calls for help (1), should provide help even if in bad mood (1). Indeed, the most activated behaviour is b_Help (1.8). A disagreeable character (1) in a neutral situation (1) and somewhat bad mood (0.5) should still be mean. The outputs are disagreeable behaviours, b_Insult (1.74), followed by b_Assault (1.51). A highly neurotic personality (1) in a stressful situation (1) and a bad mood (1) should produce emotionally unstable behaviours. Appropriately, the model output is a cry behaviour (2.86). A person who is very open to experience (1), when put in a situation which calls for exploration (1) should explore even if in bad mood (1). Indeed, the explore behaviour is most activated (2.24). Finally, someone who is very conscientious (1), when placed in a situation demanding persistence at some task (1), should persist despite a bad mood (1). The model result is correspondingly b_Persist (1.75).

\subsection{Different personalities do things differently in the same situation}

To show that SPOT produces different and interesting personality-appropriate behaviours, we need to show that different personalities react differently to the same situation. We will choose a neutral situation (0.75) and assign a neutral mood $(0.75)$ for each personality. Table 3 summarizes the results. If we include the cry output behaviour, then there are five different behaviours that are produced in this

Table 2. Input personality, situation and mood values, along with corresponding output behaviours, demonstrating personality-appropriate behaviours for the big five traits.

\begin{tabular}{llll}
\hline Personality & Input situation & Input mood & \multicolumn{1}{c}{ Output behaviour } \\
\hline Extrovert (1) & Neutral (1) & Neutral (1) & Talk (2.14) \\
Introvert (1) & Neutral (1) & Neutral (1) & None (2.7), Avoid (1.6) \\
Agreeable (1) & Help (1) & Bad (1) & Help (1.80) \\
Disagreeable (1) & Neutral (1) & Bad (0.5) & Insult (1.74), Assault (1.51) \\
Neurotic (1) & Stress (1) & Bad (1) & Cry (2.86) \\
Open (1) & Explore (1) & Bad (1) & Explore (2.24) \\
Conscientious (1) & Persist (1) & Bad (1) & Persist (1.75)
\end{tabular}


Table 3. Input personality and output behaviour activation values for a neutral situation (0.75) and a neutral mood (0.75).

\begin{tabular}{ll}
\hline Personality & Output behaviour \\
\hline Extrovert (1) & Talk (1.9) \\
Introvert (1) & None (2.32) \\
Agreeable (1) & Talk (1.65) \\
Disagreeable (1) & Insult (1.4) \\
Neurotic (1) & None (1.51), Cry (1.5) \\
Open (1) & Talk (1.52) \\
Conscientious (1) & None (1.66) \\
Disagreeable (0.8), Neurotic (0.8) & Avoid (1.52) \\
\hline
\end{tabular}

situation, depending on the personality being modelled. The behaviours are furthermore appropriate for each personality and also appropriate for the situation and mood.

\subsection{Simulating personality change}

To illustrate how personality and behaviour changes in SPOT, we will pick a few characters and expose them to some environmental situations, which will change their personalities. The resulting behaviour will be compared with the initial behaviour in the same situation, before personality changed.

Friendly situations in SPOT make people more agreeable and extroverted, and at the same time less disagreeable and introverted. Let us pick a character who is fairly introverted (0.75) and disagreeable $(0.75)$ to start off with, and also slightly extroverted (0.2) and agreeable (0.2). Their behaviour in a neutral situation (0.5) and in neutral mood (0.5) is b_None (1.7), followed by b_Avoid (1.51). After encountering five friendly situations, this person's personality becomes 0.5 introverted and disagreeable, and 0.45 extroverted and agreeable. This time in the same original neutral situation (0.5) and in neutral mood (0.5), this person chooses to talk (1.55), followed by doing nothing (1.48). This change reflects their new more social and nicer personality, due to experiencing friendly situations.

Stressful situations make people more neurotic. A somewhat conscientious (0.5) and neurotic (0.5) personality in a fairly hostile situation (0.7) and in fairly bad mood (0.7) will choose to avoid (1.98), followed by crying (1.84). After exposure to five stressful situations, this person becomes 0.75 neurotic and still 0.5 conscientious. When placed in the same hostile situation (0.7) and in bad mood (0.7), this character displays neurotic behaviour and cries (2.21), followed by avoiding (2.15).

Personality change in the model as a result of environmental experiences can be considered to be learning by the character about different situations and their consequences. To illustrate this let us look at a character who is highly extroverted $(0.8)$ to begin with. When placed in a fairly hostile situation (0.9) but in quite good mood (0.75), this character tries to deal with the situation by talking (1.32), although the avoid behaviour is a close second choice (1.22). We can view the character as being gullible or too trusting, due to his extroverted nature and good mood. However, this hostile encounter changes his personality to 0.75 extroverted and 
0.005 introverted. The second time in the same hostile (0.9) situation and in good mood (0.75), this person still chooses to talk (1.26), but with lesser strength. Again, his personality changes as a result of the hostile encounter to 0.7 extroverted, 0.005 introverted, and 0.1 disagreeable. The next time in the same original hostile (0.9) situation and in the same good mood (0.75), this character will avoid (1.23), followed by insulting (1.21) and talking (1.20) as a third choice. Thus, in only two hostile experiences, this person has learned not to be so gullible and trusting in future hostile situations. Such learning or personality change is both psychologically plausible and very interesting as a way of varying video game characters' behaviours over time.

\subsection{Evaluation and comparison of results}

We will now evaluate SPOT based on the three criteria of a good computational personality model given in the introduction. As listed in section 1.6 these criteria are: (1) psychological plausibility and realism, (2) simplicity and efficiency, and (3) interesting and varied model behaviour.

SPOT has numerous psychologically-inspired components. First of all, it covers three major personality theories to some extent. It is based primarily on the Big Five trait theory, represented in the model by the personality trait nodes and their connections to behaviours corresponding to those traits. All five of the OCEAN traits are modelled, and any combination of these traits is possible. SPOT also adheres to the Social-Learning theories, since particular situations can influence behaviour choices and personality. Finally, the initial specification of the personality node values can be considered giving the modelled characters genetic predispositions for behaviours corresponding to those traits, in accordance with Psychobiological personality theories.

SPOT also has moods and emotions, which are determined by personality and situations. As a significant new addition personality change or development over time is modelled. Personality change is influenced by the environment, and is based on psychological findings that suggest how the environment might shape personality.

One of the main positive features of our model is simplicity and efficiency. The design is very straightforward, and can be easily modified. For instance, if a new node is required (a new input situation or output behaviour), one just has to add that node to the network and set up its corresponding connections to the other existing nodes - the rest of the network remains unchanged. However, new model simulations involving existing nodes that are affected by connections from the newly added node will be required, to make sure that the added node and its connections do not adversely affect the existing connections. In general, 'fine-tuning' the connection weights to validate model behaviour required many simulations and was time-consuming.

Specification of a personality and inputs in SPOT is trivial: one simply assigns values to the desired nodes. Execution time is constant: activation flows feedforward through three (fixed) network layers with the simplest possible activation function and no settling is required. Memory consumption is also minimal: only one copy of the network needs to be stored in memory, and seven decimal values for the specification of each character's personality. The personality change program is less than 200 lines of code, executes in constant time and only one copy is required in memory. This simplicity and efficiency is extremely important for video games, 
as with the current model we can have hundreds or thousands of modelled characters interacting in the game in real-time.

Currently SPOT has nine possible output behaviours, allowing for a fair degree of action choices. Additional output behaviours can easily be specified if necessary. A modelled character's behaviour is furthermore interesting because it is the outcome of three interacting components: personality, situation and moods/ emotions. All these factors are taken into consideration when producing an output behaviour for any input situation. A character reacts differently to the same situation depending on his or her personality as well as mood.

Most importantly, the new personality change component of SPOT allows for more interesting and varied character behaviour over time. As a simulated character's personality changes due to encountering different situations throughout the game or simulation, his or her behaviours also change. This means that the same character, in the exact same situation and mood, might respond differently at two separate times. Moreover, the behaviour variability is psychologically plausible and results from actual situations encountered in the game, as opposed to random variability for example.

SPOT is ideally suited for a Sims-type video game. Specification of a character's personality is extremely simple, and one can choose any combination of the Big Five personality traits, allowing for a vast range of different personalities. Characters have a choice of three possible moods they can be in, and they can respond to numerous situations as well as other characters. Characters also have a good variety of possible output behaviours to choose from. Additional inputs and outputs can be easily included as explained above. Very fast model execution and minimal memory consumption is another huge asset for a video game application. Finally, the addition of personality change will create characters whose personalities and behaviours develop as the game progresses, resulting in more complex, entertaining and believable interactions.

\section{Conclusion}

This article presents a new computational model of personality, SPOT (simulating personality over time), that includes personality change. SPOT is psychologically based and intended to be used as an application in a Sims-type video game. SPOT has four basic components: personality, emotions, inputs describing the situations and behaviour outputs. Personality in SPOT is based on behavioural tendencies of the Big Five trait model, and allows one to specify a personality consisting of any combination of the Big Five traits. Six basic emotions are modelled in SPOT: neutral, sad, happy, angry, afraid and curious. The personality of a character and his or her behaviour results from the interplay of three components: personality traits, emotions (and moods) and situational factors. Environmental situations shape personality change over time in SPOT. Simple, psychologically inspired rules determine which situations change personality and in what ways.

Personality and behaviour modelling are becoming increasingly more important in many fields of computer science and psychology. Future work on more complex and realistic computer personality models will help us understand personality better and create more human-like virtual characters. 


\section{Acknowledgements}

This research was supported by the Natural Sciences and Engineering Research Council of Canada.

\section{References}

E. Andre, M. Klesen, P. Gebhard, S. Allen and T. Rist, "Integrating models of personality and emotions into lifelike characters", in Proceedings of the workshop on Affect in Interactions Towards a new Generation of Interfaces in conjunction with the 3rd i3 Annual Conference, A. Paiva and C. Martinho Eds., Siena, Italy, 1999, pp. 136-149.

E. Andre, M. Klesen, P. Gebhard, S. Allen and T. Rist, "Exploiting models of personality and emotions to control the behavior of animated interactive agents". Technical Report, DFKI GmbH, Saarbrucken, Germany, 2000 (www.dfki.de).

J. Bates, A.B. Loyall and W.S. Reilly, "An architecture for action, emotion and social behavior". Technical Report CMU-CS-92-144, School of Computer Science, Carnegie Mellon University, Pittsburgh, PA, 1992.

N.R. Carlson and W. Buskist. Psychology: The Science of Behavior, 5th Edn, Boston, MA: Allyn \& Bacon, 1997.

H.J. Eysenck and S. Rachman. The Causes and Cures of Neuroses, London: Routledge and Kegan Paul, 1965.

N. Frijda. The Emotions. Studies in Emotion and Social Interaction, New York: Cambridge University Press, 1986.

T.F. Heatherton and J.L. Weinberger (Eds), Can Personality Change?, Washington, DC: American Psychological Association, 1994.

JavaNNS, “Java Neural Network Simulator", University of Tubingen, July 2003. Available online at: http://www-ra.informatik.uni-tuebingen.de/software/JavaNNS/welcome_e.html

L. Kaufman and P.J. Rousseeuw. Finding groups in data: An introduction to cluster analysis, New York: Wiley, 1990.

B. King, "Games started off without a bang", Wired News, 15 July 2002. Available online at: http:// www.wired.com/news/games/0,2101,53765,00.html

S. Kshirsagar and N. Magnenat-Thalmann, "A multilayer personality model", in Proceedings of 2nd International Symposium on Smart Graphics, June 2002, pp. 107-115.

J.E. Laird and M. van Lent, "Human-level AI's killer application: interactive computer games", AI Magazine, 22(2): 15-25, 2001.

P.J. Lang, The Emotion Probe: Studies of Motivation and Attention, A Study in the Neuroscience of Love and Hate, Hillsdale, NL: Lawrence Erlbaum Associates, Publishers, 1995.

J.C. Loehlin, Genes and Environment in Personality Development, London: Sage Publications, 1992.

A.B. Loyall and J. Bates, "Personality-rich believable agents that use language", in Proceedings of the First International Conference on Autonomous Agents, February 1997, Marina del Rey, CA.

B. Mac Namee and P. Cunningham, "The $\mu$-SIC system: a connectionist driven simulation of socially interactive agentsr". Technical Report, Computer Science Department, University of Dublin (Trinity College), 2002.

D. Moffat, "Personality parameters and programs", in Creating Personalities for Synthetic Actors: Towards Autonomous Personality Agents, R. Trappl and P. Petta, Eds., Berlin: Springer, 1997.

J. Nerb and H. Spada, "Evaluation of environmental problems: a coherence model of cognition and emotion", Cognition and Emotion, 15, pp. 521-551, 2001.

A. Ortony, G. Clore and A. Collins, The Cognitive Structure of Emotions, Cambridge: Cambridge University Press, 1988.

S.V. Paunonen, "Big five factors of personality and replicated predictions of behavior", Journal of Personality and Social Psychology, 84(2), pp. 411-424, 2003.

L.A. Pervin, Personality: Theory and Research, 5th Edn, New York: John Wiley \& Sons, Inc, 1989.

S.J. Read and L.C. Miller, "Virtual personalities: a neural network model of personality", Personality and Social Psychology Review, 6(4), pp. 357-369, 2002.

P. Rizzo, A. Cesta and M. Miceli, "Personality-driven believable agents for interactive entertainment". Technical Report, Institute of Psychology of the National Research Council (IP-CNR), Rome, Italy, 1998. Available online at: http://aos2.uniba.it:8080/napoli/rizzo.htm

D. Rousseau and B. Hayes-Roth, "Personality in synthetic agents". Technical Report No. KSL 96-21, Knowledge Systems Laboratory, Department of Computer Science, Stanford University, Stanford, CA, 1996. 
D. Rousseau and B. Hayes-Roth, "A social-psychological model for synthetic actors". Technical report no. KSL 97-07, Knowledge Systems Laboratory, Department of Computer Science, Stanford University, Stanford, CA, 1997.

Y. Shoda and W. Mischel, "Personality as a stable cognitive-affective activation network: characteristic patterns of behavior variation emerge from a stable personality structure", in Connectionist Models of Social Reasoning and Social Behavior, S.J. Read and L.C. Miller, Eds., Hillsdale, NL: Lawrence Erlbaum Associates, Inc., 1998.

Y. Shoda, S. LeeTiernan and W. Mischel, "Personality as a dynamical system: emergence of stability and distinctiveness from intra- and interpersonal interactions", Personality and Social Psychology Review, 6(4), pp. 316-325, 2002.

Stuttgart Neural Network Simulator (SNNS), 2003. University of Stuttgart and University of Tubingen. Available online at: http://www-ra.informatik.uni-tuebingen.de/SNNS/

S. Srivastava, O.P. John, S.D. Gosling and J. Potter, "Development of personality in early and middle adulthood: set like plaster or persistent change?", Journal of Personality and Social Psychology, 84(5), pp. 1041-1053, 2003.

P. Thagard, "Why wasn't OJ convicted? Emotional coherence in legal inference", Cognition and Emotion, 17, pp. 361-383, 2003.

"The Sims Becomes The Best Selling PC Game of All Time", GameZone News, 21 March 2002. Available online at: http://www.gamezone.com/news/03_21_02_01_02PM.htm

R. Trappl and P. Petta, Creating Personalities for Synthetic Actors: Towards Autonomous Personality Agents, Berlin: Springer, 1997.

P.A. Vernon, K.L. Jang, J.A. Harris and J.M. McCarthy, "Environmental predictors of personality differences: a twin and sibling study", J. Personality and Social Psychology, 72(1), pp. 177-183, 1997.

B. Wagar and P. Thagard, "Spiking Phineas Gage: a neurocomputational theory of cognitive-affective integration in decision making", Psychological Review, 111(1), pp. 67-79, 2004.

M. Wish, M. Deutsch and S. Kaplan, "Perceived dimensions of interpersonal relations", J. Personality and Social Psychology, 33(6), pp. 409-420, 1976. 